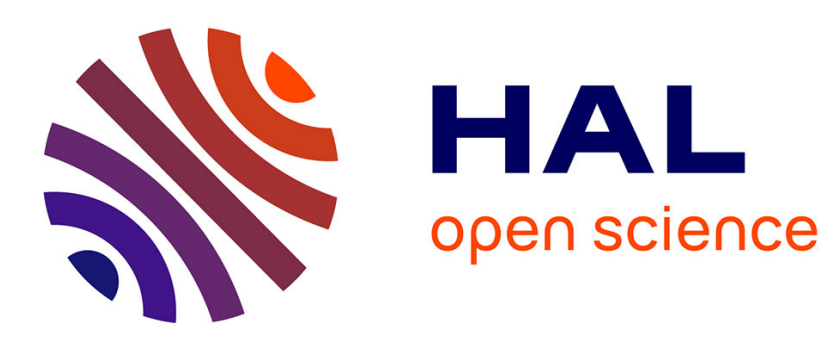

\title{
Direct writing of piezoresistive silicon resistors using laser-induced CVD
}

\author{
H. Moilanen, L. Leppävuori, A. Uusimäki
}

\section{To cite this version:}

H. Moilanen, L. Leppävuori, A. Uusimäki. Direct writing of piezoresistive silicon resistors using laser-induced CVD. Journal de Physique IV Proceedings, 1993, 03 (C3), pp.C3-457-C3-464. 10.1051/jp4:1993363 . jpa-00251420

\section{HAL Id: jpa-00251420 https://hal.science/jpa-00251420}

Submitted on 1 Jan 1993

HAL is a multi-disciplinary open access archive for the deposit and dissemination of scientific research documents, whether they are published or not. The documents may come from teaching and research institutions in France or abroad, or from public or private research centers.
L'archive ouverte pluridisciplinaire HAL, est destinée au dépôt et à la diffusion de documents scientifiques de niveau recherche, publiés ou non, émanant des établissements d'enseignement et de recherche français ou étrangers, des laboratoires publics ou privés. 


\title{
Direct writing of piezoresistive silicon resistors using laser-induced CVD
}

\author{
H. MOILANEN, L. LEPPÄVUORI and A. UUSIMÄKI \\ Microelectronics and Material Physics Laboratories, University of Oulu, Linnanmaa, 90570 Oulu, Finland
}

\begin{abstract}
Laser-induced chemical vapour deposition (LCVD), using a movable focused $\mathrm{Ar}^{+}$laser beam $(1 \mathrm{~W}, 514.5 \mathrm{~nm})$, was used for direct, maskless writing of strain sensitive silicon resistors for the fabrication of prototype pressure sensors on a silicon on sapphire (SOS) and sapphire substrates from the mixed ratio of $0.1 \% \mathrm{~B}_{2} \mathrm{H}_{6}$ in the reactive gas $\mathrm{SiH}_{4}$. The strain sensitive resistors, approximately $300 \mu \mathrm{m}$ long, were deposited, with in situ measurement of their resistance. The width of the deposited stripes were about $10 \mu \mathrm{m}$, and lines over $1 \mu \mathrm{m}$ thick were written at speeds of $50 \mu \mathrm{m} / \mathrm{s}$. The resistivity of the stripes was $7-10 \mathrm{~m} \Omega \mathrm{cm}$. The LCVD processing pressure was typically $100 \mathrm{mbar}$. In the case of silicon-on-sapphire (SOS) diaphragms (thickness of $330 \mu \mathrm{m}$ ) longitudinal gauge factors of $40-80$ were observed when the direction of the resistors was parallel to the [110]-type epitaxial direction. The gauge factor of 13 was measured when the resistor was deposited parallel to the [100]-type direction. Polycrystalline deposition on the sapphire substrate (thickness of $530 \mu \mathrm{m}$ ) gave gauge factors of $31-48$. Temperature coefficients of resistance $\left(0^{\circ} \mathrm{C} . .70^{\circ} \mathrm{C}\right)$ of silicon stripes were $-500 \ldots-$ 2200 (SOS) and $-2300 \ldots-2900 \mathrm{ppm} /{ }^{\circ} \mathrm{C}$ (sapphire), respectively.
\end{abstract}

\section{Introduction.}

The laser-induced CVD of silicon stripes from silane with a scanning laser beam has been the subject of many studies since 1980 . The areas where the flexibility of the chemical laser processing could have real advantages are direct writing of chip conductor lines, making connections for packaging (chip wiring), trimming and customisation of circuits in integrated sensors, fabricating of micro-mechanical structures and processing of optoelectronic components. An other possible field of applications where the flexibility of the laser writing of stripes of various materials may find applications is the sensor technology. Further more, the use of lasers to manufacture small-scale production sensors can considerably reduce the turnaround time.

A laser beam can be used to activate a reaction at a gas/solid interface by excitation of the gas phase or gas molecules which are adsorbed on to the surface of the solid. Various gaseous, liquid and solid reactants have been used to deposit a variety of metals, semiconductors and insulators, as well as compound materials [1]. A number of physical processes are involved which include the absorption of laser energy, control of reaction rates, initiation of product nucleation and adsorption or desorption of molecules on the surface. The spatial confinement of the laser beam leads to the confinement of the reaction to micrometer 
dimensions on the surface. It has been shown that the high degree of spatial control associated with this form of laser stimulated surface chemistry can be the basis of direct, maskless, fabrication techniques for microelectronics [2]. Such techniques can be based either on photochemical or thermal driving of chemical reactions. Photochemical techniques are intrinsically more efficient in their use of laser energy and have the advantage of the surface temperature rise being negligible. Surface thermal techniques are less prone to interference from the onset of unwanted homogeneous reactions which could cause effective enlargement of the reaction zone into the gas or liquid phase above substrate. However, very high processing speeds up to several $\mathrm{mm} / \mathrm{s}$ are possible for thermal deposition reactions.

We have studied the thermal deposition of silicon stripes on insulating substrates using $\mathrm{Ar}^{+}$laser and analyzed their piezoresistivity properties. Because the piezoresistivity in a semiconductor is a highly anisotropic property, the applications would preferably require stripes having single crystalline structure with controlled orientation on a substrate. In this study, LCVD, using a movable focused $\mathrm{Ar}^{+}$laser beam, was used for direct, maskless writing of strain sensitive silicon resistors for the fabrication of prototype pressure sensors on silicon-on-sapphire (SOS) and sapphire substrates from the mixed ratio of $0.1 \%$ diborane $\left(\mathrm{B}_{2} \mathrm{H}_{6}\right)$ in the reactive gas silane $\left(\mathrm{SiH}_{4}\right)$. In $\mathrm{SOS}$ layered structures the thin silicon layer increases considerably the absorption of the laser radiation which could make the laser-induced deposition easier than in the case of pure sapphire. The stripes deposited on SOS substrates had a anisotropic single crystalline silicon characteristics whilst the stripes deposited directly on sapphire had a polycrystalline structure.

\section{Piezoresistivity conditions in p-type Si.}

The piezoresistivity is a property closely related to the band structure of the charge carriers in the semiconductor. The relative change in the resistivity due to elastic strain (the gauge factor) is, therefore, a highly anisotropic property with respect to crystalline directions. In the case of longitudinal gauge factor the elongation takes place parallel to the current flow whereas for the transversal gauge factor the elongation is perpendicular to the current. In a p-type silicon, the longitudinal gauge factor is largest in the [111]-type direction, whereas, in the [100]-type direction, the gauge factor is very small. In practice, p-type silicon resistors, along an [110]-type direction where the gauge factor has its maximum value on an (100)-surface, are most often used for piezoresistive sensor applications. The epitaxial crystalline orientation of the silicon. stripes, in interest, can be grown on typically used (1102) sapphire substrates. The both [2021]- and [1101]-type directions of the sapphire coincide with the [110]- and [100]-type directions of epitaxial silicon. The transversal gauge factor also has a strong orientation dependence in a single crystalline semiconductor. In a polycrystalline semiconductor the gauge factor is usually smaller than in a single crystalline material because a random polycrystalline material includes plenty of crystallites having an unfavourable orientation with respect to the strain and current directions. The potential barriers at the grain boundaries also have an effect on the piezoresistivity, especially at low doping concentrations and small grain sizes [3].

\section{LCVD equipment.}

The laser beam is focused through a $20 \mathrm{~mm}$ focal length lens which can be moved over an area of $100 \times 100$ $\mathrm{mm}^{2}$ with a resolution of $80 \mathrm{~nm}$, either manually or via a microcomputer, by two perpendicularly mounted translation stages driven by step motors. The accuracy of positioning the beam is about $\pm 1 \mu \mathrm{m}$. The facility allows the monitoring of the work piece through a microscope eyepiece and, during processing, with a $\mathrm{CCD}$ camera and monitor. The movements of the translation stages and the synchronous triggering of the pulses of the lasers are controlled by a microcomputer. The apparatus for laser-induced chemical vapour deposition (LCVD) consists of an Ar+ laser, a gas cabinet with an arrangement for automatically purging the silane/diborane gas, a reaction chamber $(0.4 \mathrm{l}$, static system), a turbomolecular pump and a thermal decomposition oven for the exhaust gases. The pressure of the process gas can be regulated up to 1 bar. 
The laser equipment is installed in a clean room of class 10000 , the area over the laser being of class 100 . Using green radiation $\left(\lambda=514.5 \mathrm{~nm}\right.$ ) from from the $\mathrm{Ar}^{+}$laser, a minimum focus diameter of $6 \mu \mathrm{m}$ can be attained. For deposition of doped silicon conductors, specially prepared mixtures of silane and diborane are used. A toxic gas alarm system continuously registers the quantity of toxic gases present during processing.

\section{Piezoresistivity measurements.}

Before the direct writing process, the diaphragm of either pure sapphire or SOS was joined by an $\mathrm{Ag}-\mathrm{Cu}$ eutectic to the titanium frame of the sensor. A circular hole with a diameter of $7.2 \mathrm{~mm}$ in the frame acted as the point of transmission of the pressure required to bend the diaphragm. An aluminium contact pattern, made using a metal mask, was evaporated on to the diaphragm. The contact pads in the sensor bridge were then wire-bonded to the corresponding pads in a plastic piece joined to the frame. Fig. 1 shows the structure and the bending behaviour of the diaphragm pressure sensor. Piezoresistivity effect was measured on the bending diaphragm, where 24 bar pressure caused a strain up to $0.5 \times 10^{-3}$.

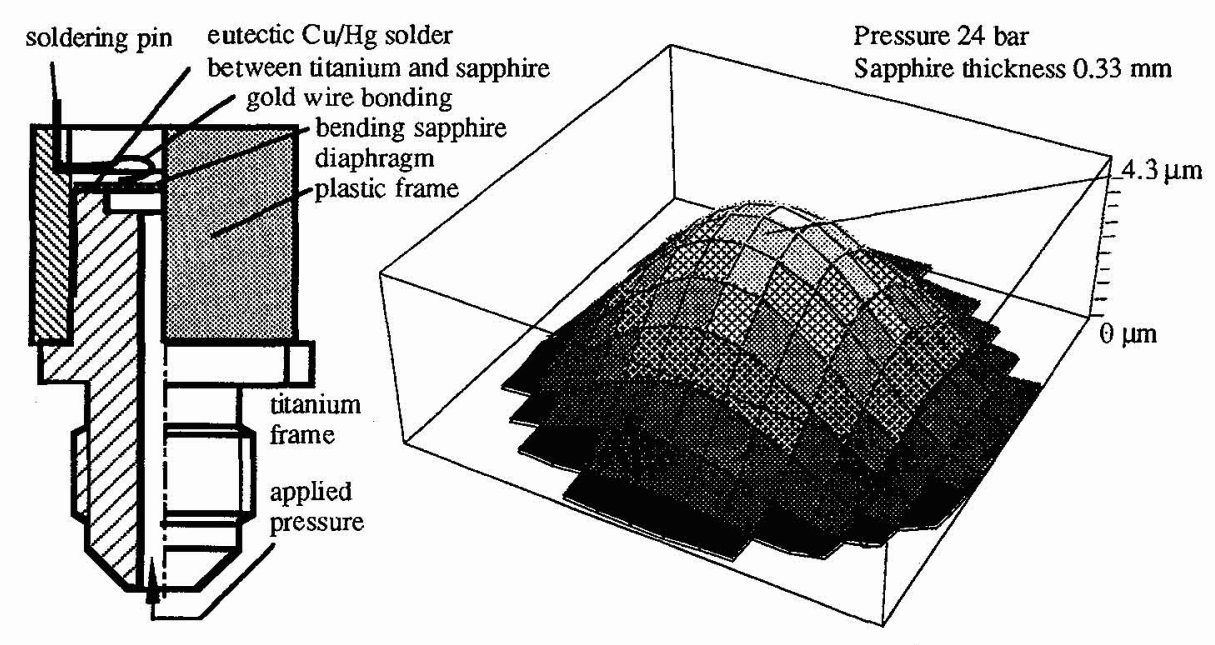

Fig. 1. - The structure and the bending behaviour of the diaphragm pressure sensor.

The strain sensitive resistors, approximately $300 \mu \mathrm{m}$ long, were then deposited on selected points of the diaphragm. The pattern of aluminium conductors allowed different possibilities for location of the four resistors of the bridge. The possibility of in situ measurement of the resistances of the deposited lines turned out to be very useful in choosing the processing parameters and the laser paths so that an approximate balance in the bridge could be attained. In all the test structures, two of the resistors were deposited radially at the edges of the diaphragm where the radial compressive strain has its maximum value and the other two were deposited either at the centre of the diaphragm or tangentially to its edges. This allowed the measurements of both longitudinal and transversal piezoresistivity in one sample. Fig. 2 shows the conductor pattern of the pressure sensor. 

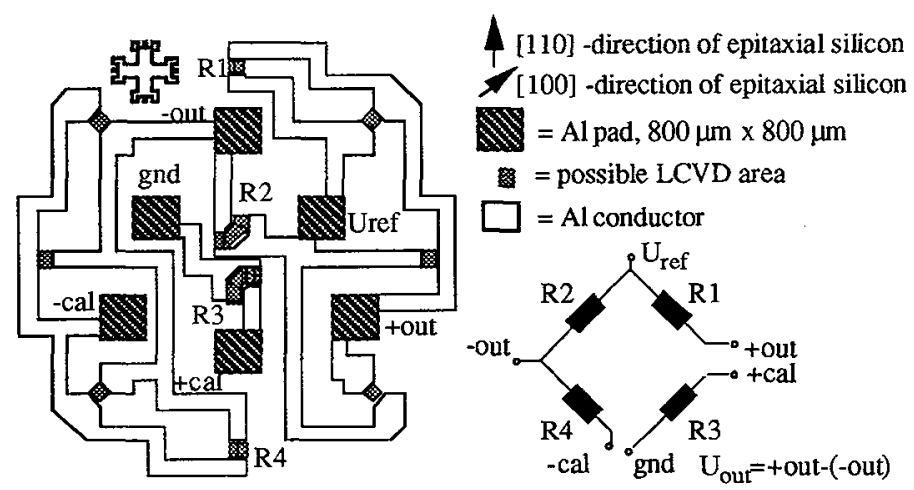

Fig. 2. - Conductor pattern of the pressure sensor (SOS).

\section{LCVD processing parameters.}

Pyrolytic decomposition requires a considerable rise in temperature in order to start the reaction on a practical scale. The growth process is therefore strongly dependent on the absorption of light in both the substrate and the deposit. In practice, the growth can be initiated at places where strongly absorbing material has been deposited by other methods, or at occasional impurity particles on the substrate. When growth has been initiated, silicon stripes can also be deposited on a weakly absorbing substrate, because the absorption of light in the growing deposit can maintain the temperature at an adequate level. The limiting factor for the linear growth rate (scanning speed) is then the deposition rate of silicon on silicon. A silane/diborane gas mixture of having a total pressure of $100 \mathrm{mbar}$ was used as a reactant gas in a reactor chamber (static system). The SOS substrates were better suited for direct writing because the existing silicon layer on the surface absorbed the laser light and caused better controlled temperature distribution to drive the decomposition reaction, $\mathrm{SiH}_{4} \longrightarrow \mathrm{Si}+\mathrm{H}_{2}$.

SEM micrographs of the deposited silicon stripes on the sapphire and SOS substrates are shown in Fig. 3. An $\mathrm{Ar}^{+}$laser with the visible $\mathrm{cw}$ radiation was used to deposit doped p-type silicon stripes on (1102) sapphire and SOS substrates. In the case SOS, the substrate is covered with a $1 \mu \mathrm{m}$ thick epitaxial silicon layer. The width of the deposited stripes were about $10 \mu \mathrm{m}$, and lines over $1 \mu \mathrm{m}$ thick were written at speeds of $50 \mu \mathrm{m} / \mathrm{s}$. Using the multimode radiation of the laser, a power of $1.0 \mathrm{~W}$ (focal length $20 \mathrm{~mm}$ ) was used for deposition. According to x-ray diffraction studies, the stripes on sapphire were found to be polycrystalline with almost random orientation. The low absorption of light in transparent substrates makes also the continuation of the growth rather critical for the growth parameters. Three different morphologies of deposited stripes were obtained. At the beginning the stripe usually has a regular geometry but a hairy surface structure. This type of stripe morphology however do not seem to be stable in a continued stripe deposition but it changes to a morphology having a more irregular geometry but a smoother surface structure. With increasing scanning speed the growth becomes more irregular leading to a grainy structure first in the center of the stripe and with still increasing speed also at the edges of the stripe.

The dimensions, as well as the morphology of the deposited lines, depended strongly on the power applied. At the lower limit of the power range, narrow lines with approximately Gaussian cross-section were obtained. With increasing power, the top of the line first became flat. With still further increase of power the lines became volcano-like shaped with a dip at the centre line. The appearance of flat profiles seemed to be connected with the existence of a molten phase during the deposition. The ripples perpendicular to the polarisation of the laser radiation could be seen in most of the lines. 


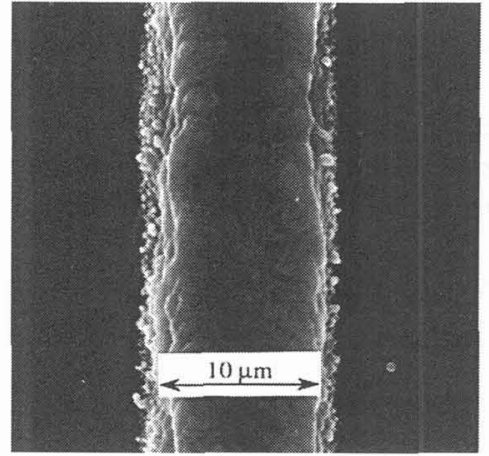

(a)

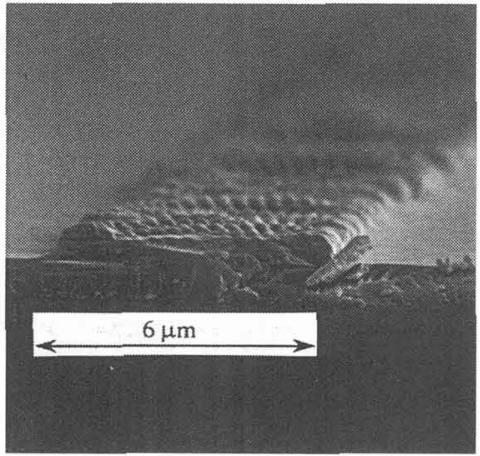

(b)

Fig. 3. - SEM micrographs of the deposited silicon stripes on the sapphire (a) and SOS (b) substrates.

5.1 LCVD OF SILICON ON PURE SAPPHIRE. - For the studies of the piezoresistivity, stripes of silicon were deposited by LCVD on sapphire substrates. First experiments with Nd:YAG laser $(\lambda=1064 \mathrm{~nm})$ using undoped silane as a reactive gas the deposited stripes were found to be of n-type with the resistivity in the range of $1 \times 10^{-2}$ to $4 \times 10^{-3} \Omega \mathrm{cm}$. In further experiments - to attain p-type silicon stripes - a mixture of $\mathrm{SiH}_{4}$ and $1 \% \mathrm{~B}_{2} \mathrm{H}_{6}$ with the total pressure in the range 100 mbar was used as reactive gas atmosphere in a reaction chamber [2]. The square resistance of the stripes varied between 1.5 and $20 \Omega$ depending on their morphology. The resistivity was in the range $2 \times 10^{-4} \ldots 1 \times 10^{-3} \Omega \mathrm{cm}$ which corresponded to doping concentrations between $1.3 \times 10^{20}$ and $6 \times 10^{20} \mathrm{~cm}^{-3}$. Results of the experiments to study the possible epitaxial crystalline orientation of the silicon stripes on sapphire substrates showed that the longitudinal gauge factors for both types of [110]- and [100]-type directions were equal. It was therefore clear that the silicon stripes deposited on sapphire do not adopt the epitaxial crystalline orientation with respect to the sapphire substrate but they had a polycrystalline character. This relatively high doping concentration regarding the piezoresistivity applications - showed that much lower boron content would be preferable. In a single crystalline p-type silicon with a boron content of $2 \times 10^{19} \mathrm{~cm}^{-3}$ the gauge factor along the [110]-type direction is about 60 which is 4 to 6 times larger than in samples made from gas mixture $\mathrm{SiH}_{4} / 1 \% \mathrm{~B}_{2} \mathrm{H}_{6}$. The difference is caused by the polycrystalline structure of the stripes and by the high doping concentration. In fact, the present measurements were in good accordance with the results given in the literature for gauge factors in highly doped polycrystalline p-type silicon [3]. In LCVD processing of the prototype pressure sensors, with a new chosen gas mixture $(0.1 \%$ diborane) which concluded to lower boron content, higher gauge factors, as shown later on, were obtained.

5.2 LCVD OF SILICON ON SOS. - The stripes deposited along various directions on the substrate revealed anisotropic behaviour in their piezoresistivity properties. The results showed that the stripes tend to adopt the same epitaxial crystalline orientation as the SOS layer. The resistivity of the stripes depended on the mixed ratio of $\mathrm{SiH}_{4}$ and $\mathrm{B}_{2} \mathrm{H}_{6}$ in the reactive gas. With $1 \% \mathrm{~B}_{2} \mathrm{H}_{6}$, the average resistivity was about 1 $\mathrm{m} \Omega \mathrm{cm}$ while with $0.1 \% \mathrm{~B}_{2} \mathrm{H}_{6}$, the resistivity of the stripes was $7-10 \mathrm{~m} \Omega \mathrm{cm}$ which corresponded to doping concentrations between $1.3 \times 10^{18}$ and $1.8 \times 10^{18} \mathrm{~cm}^{-3}$.

\section{Properties of piezoresistivity.}

In the case of SOS diaphragms (thickness of $330 \mu \mathrm{m}$ ) the longitudinal gauge factor $\mathrm{GF}_{\mathrm{L}}$ of $40-80$ were observed when the direction of the resistors was parallel to the [110] type epitaxial direction (shown in Fig. 5.). The gauge factor of 13 were measured when the resistor was deposited parallel to the [100] type 
direction. Polycrystalline deposition on the sapphire substrate (thickness of $530 \mu \mathrm{m}$ ) gave gauge factors of 31-48. The temperature coefficients of resistance $\left(0^{\circ} \mathrm{C} . . .70^{\circ} \mathrm{C}\right)$ of silicon stripes - effected by the titanium frame - were $-500 \ldots-2200$ (SOS) and $-2300 \ldots-2900 \mathrm{ppm} /{ }^{\circ} \mathrm{C}$ (sapphire). Results are given in Fig. 6. The lines deposited on SOS substrates along the different crystalline directions showed clear anisotropy in their gauge factors. The character of the isotropy was as expected like that of non-epitaxial p-type silicon on sapphire.

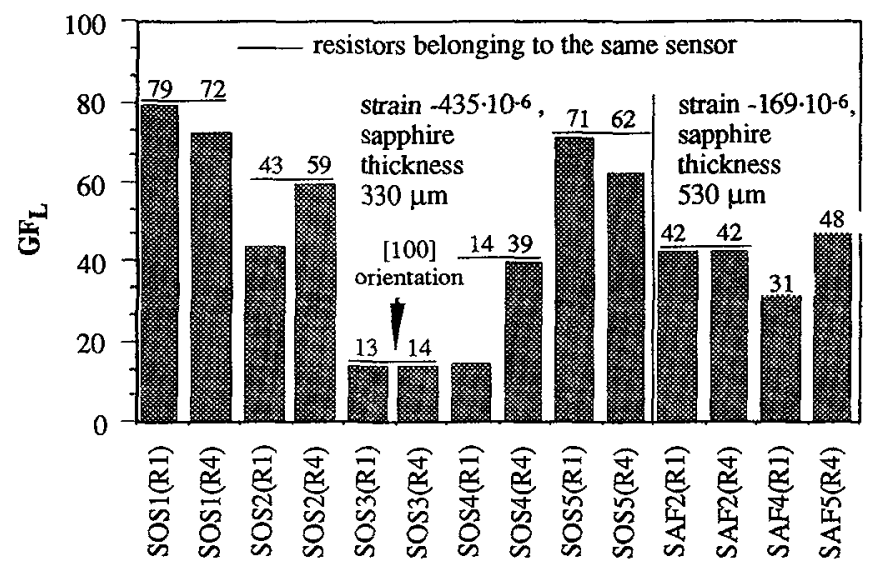

Fig. 5. - The longitudinal gauge factors of SOS and sapphire (SAF) pressure sensors.

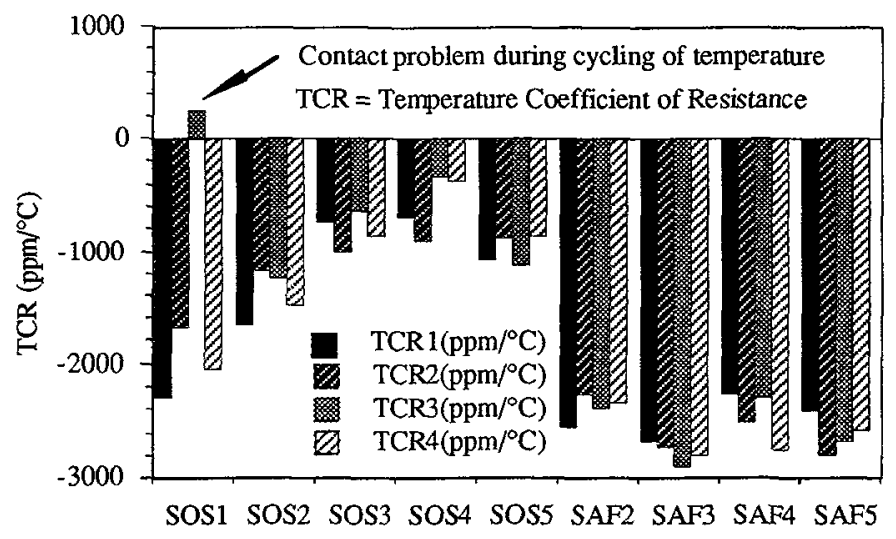

Fig. 6. - The temperature coefficients of resistances of SOS and sapphire pressure sensors.

\section{Discussion.}

If the maximum temperature produced by the laser beam exceeds the melting point of silicon the deposited stripe becomes broader and gets a volcanolike profile with a dip at the center line. According to the numerical simulations the peak temperature is a steep function of both the incident laser power and of the spot diameter [4]. Because a decreasing spot diameter results to an increasing peak temperature, it is possible that in some special cases the decreasing spot diameter causes broadening of the deposited stripe. This is the case when the peak temperature reaches the melting point of silicon. At the melted phase, silicon can easily flow out of the beam area. The morphology of the stripes does not differ essentially from those 
deposited on silicon or on glass covered by thin silicon layer but there is a great difference in morphology compared with pure sapphire substrates. The low absorption of light in pure sapphire makes the nucleation much more difficult and the growth more irregular than in SOS.

The epitaxial character of the stripes seems to be most pronounced when sufficient laser power (1W) is used for deposition. The results seem to confirm that a temperature near the melting point of silicon is needed to assure epitaxial deposition of silicon stripes on SOS. Polycrystalline silicon resistors grown by LPCVD (Low Pressure Chemical Vapour Deposition) give maximum longitudinal gauge factors at the doping concentration of $1.0 \times 10^{19}$ [3]. In comparison with LCVD work using sapphire substrates, both of these methods produce equal gauge factors (as shown in Fig. 7). The curves show also that the anisotropy in the gauge factors - in the case of SOS substrates - is a strong evidence towards the epitaxial structure of the stripes. The longitudinal gauge factor dependences on doping concentration (p-type, boron) using different silicon fabrication techniques can also be seen in the figure $[3,5]$.

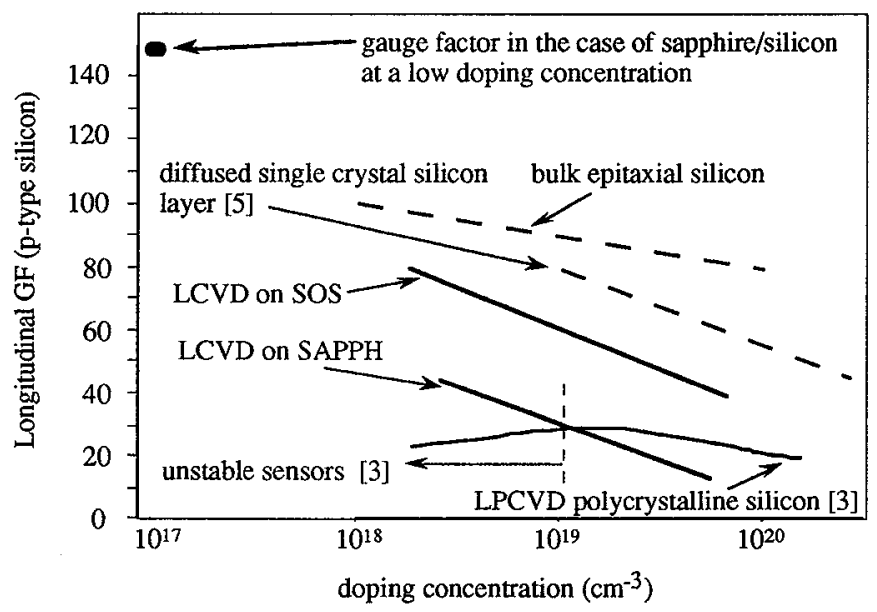

Fig. 7. - The longitudinal gauge factor dependences on doping concentration using different silicon fabrication techniques.

\section{Conclusions.}

In this study, Laser-induced chemical vapour deposition (LCVD), using a movable focused $\mathrm{Ar}^{+}$laser beam $(1 \mathrm{~W}, 514.5 \mathrm{~nm}$ ), was used for direct, maskless writing of strain sensitive silicon resistors for the fabrication of prototype pressure sensors on silicon-on-sapphire (SOS) and sapphire substrates from the mixed ratio of $0.1 \%$ diborane $\left(\mathrm{B}_{2} \mathrm{H}_{6}\right)$ in the reactive gas silane $\left(\mathrm{SiH}_{4}\right)$.

The silicon stripes deposited on sapphire do not adopt the epitaxial crystalline orientation with respect to the sapphire substrate but they have a polycrystalline character. The stripes deposited along SOS substrates revealed anisotropic behaviour in their piezoresistivity properties. In the case of SOS diaphragms (thickness of $330 \mu \mathrm{m}$ ) the longitudinal gauge factors of $40-80$ were observed when the direction of the resistors was parallel to the [110] type epitaxial direction. The gauge factor of 13 were measured when the resistor was deposited parallel to the [100] type direction. Polycrystalline deposition on the sapphire substrate (thickness of $530 \mu \mathrm{m})$ gave gauge factors of 31-48. Temperature coefficients of resistance $\left(0^{\circ} \mathrm{C} \ldots 70^{\circ} \mathrm{C}\right)$ of silicon stripes were $-500 \ldots-2200(\mathrm{SOS})$ and $-2300 \ldots-2900 \mathrm{ppm} /{ }^{\circ} \mathrm{C}$ (sapphire), respectively. 


\section{REFERENCES}

[1] BÄUERLE D., Laser-Induced Chemical Vapour Deposition, Laser Processing and Diagnostics, Springer Ser. Chem. Phys. Berlin, Heidelberg: Springer-Verlag 39 (1984) 166-182.

[2] LENKKERI J. and LEPPÄVUORI S., Laser-assisted Direct Writing of Strain Sensitive Silicon Resistors, Sensors and Actuators, A 21-23 (1990) 1011-1014.

[3] FRENCH P. and EVANS A., Piezoresistance in polysilicon and it's applications to strain gauges, Solid-State Electronics 32 (1989) 1-10.

[4] LEVOSKA J., RANTALA T.T. and LENKKERI J., Numerical simulation of temperature distributions in layered structures during laser processing, Applied Surface Science 00 (1988) ASS0110P.

[5] TUFTE O. and STELZER E., Piezoresistive Properties of Silicon Diffused Layers, J. A. Phys. 34, (1963) 313-318. 